\title{
Mass Spectrometric Imaging of Gold Nanolayer Coated Latent Fingermarks: Deciphering Overlapping Features by Statistical Analysis
}

\author{
Christian Elsner1, Bernd Abel1,2* \\ ${ }^{1}$ Leibniz Institute of Surface Modification, Leipzig, Germany \\ ${ }^{2}$ Wilhelm-Ostwald-Institute for Physical and Theoretical Chemistry, Universität Leipzig, Leipzig, Germany \\ Email: ^bernd.abel@iom-leipzig.de
}

How to cite this paper: Elsner, C. and Abel, B. (2016) Mass Spectrometric Imaging of Gold Nanolayer Coated Latent Fingermarks: Deciphering Overlapping Features by Statistical Analysis. Advances in Chemical Engineering and Science, 6, 584-599. http://dx.doi.org/10.4236/aces.2016.65050

Received: October 23, 2016

Accepted: December 25, 2016

Published: December 29, 2016

Copyright (c) 2016 by authors and Scientific Research Publishing Inc. This work is licensed under the Creative Commons Attribution International License (CC BY 4.0).

http://creativecommons.org/licenses/by/4.0/ (c) (i) Open Access

\begin{abstract}
Overlapping latent fingermarks constitute a serious challenge to database related recognition and matching algorithms in biometry, forensic and crime scene investigations. Mass spectrometry imaging (MSI) is a powerful tool for deciphering and analyzing overlapping fingermarks based on the individual chemical information of each deposit. Fingermark MSI in practice still requires a subjective judgment of an MSI expert, such that rapid analysis, automation, standardization, and a quantitative evaluation of the complete detection and separation process of overlapped fingermarks from MSI data sets is the ultimate goal and will be necessary to become an accepted process in criminal investigations and law enforcement. Here we investigated the feasibility and efficiency of different statistical approaches for the separation of overlapped latent fingermarks based on MSI data. Entropy analysis of generated $\mathrm{m} / \mathrm{z}$-images was used to evaluate the results obtained from the statistical analysis. Furthermore, we demonstrate and discuss the opportunity to reconstitute and separate overlapping fingermarks by discrete scanning at selected $\mathrm{x}, \mathrm{y}$-positions defined from a previous image analysis using a more simple schema based on visible and therefore optical distinguishable overlapped ink-based fingermarks. The overlapped latent fingermarks were developed by rapid gold sputter coating and analyzed by laser based MSI, without (organic) matrix preparation. Latent finger marks can be transferred from the substrate/surface with and conserved on a soft gold sputtered soft membrane at low temperatures.
\end{abstract}

\section{Keywords}

Overlapping Latent Fingermarks, Mass Spectrometry Imaging, Statistical Analysis 


\section{Introduction}

One of the main tasks of a forensic investigator nowadays is the visualization and collection of the topography of latent fingermarks at a crime scene location, based on reliable and cheap techniques developed in the last century [1]. This situation may significantly change in the future, if simple, transportable, fast, accurate, high-resolution, and cost-effective methods allow for the rapid topographical and in particular chemical analysis of a fingermark. The presence or absence of endogenous or exogenous compounds in a fingermark, e.g. medical or narcotic drugs, cosmetics or explosive materials may classify offenders and direct investigations in a certain direction [2] [3] [4]. Markers for age, gender, ethnic origin, disease, medication are currently under investigation [5]. New techniques are targeted on the specific detection of low abundant species, e.g., by immunological multi-metal deposition (iMMD) [6]. Thus, it is expected that the fingermark may become a renewed key role in investigative forensics, profiling, or to expand the field, even in medical diagnostics due to the opportunity of a permanent and non-invasive collection of sample material [7]. Due to the requirement of "preservation of evidence" in law-enforcement, the original topography of the fingermark has to be retained during the examination process and should not be destroyed or modified during analysis or storage. Minimal invasive imaging technologies may meet these requirements. Due to its unique ability to detect small amounts of multiple analytes in a complex matrix in a single step, without complicated pre-treatment, mass spectrometry imaging (MSI) has a large potential in fingermark analysis [8] [9] [10].

Overlapped fingermarks are frequently observed at crime scene locations and they constitute a serious challenge to recognition algorithms and matching accuracy during database queries. Thus, ongoing research is devoted to reliable methods to separate them. Full-scan mode MSI based on DESI-, MALDI-TOF- or SI-MS were employed to discriminate overlapped fingermarks [11] [12] [13] [14]. However, the long lasting procedure of scanning complete fingermarks and the large set of obtained molecular data, which have to be handled, are the main drawbacks of the MSI approaches. They not just limit applicability in forensic cases but even the study of current associated research topics such as the MS analysis of fingermarks on different substrates, their transfer during sample preparation, their conservation, and even the processing of large sample sets to obtain evidence in marker related studies. Moreover, really fast optical methods combined with smart and powerful mathematical algorithms for the separation of overlapped fingermarks are under development and in view of this the main question which has to be answered is: How can expensive mass spectrometry approaches contribute further in a competitive manner to that forensic topic [15]? Generally speaking, time and reduction of manual and subjective processes could be crucial factors for the acceptance of MSI in fingermark analysis in the future. Automated focusing on the most interesting regions of a fingermark to get a deep view inside on it and combining them with results obtained from other techniques could be an acceptable solution. Depending on the study case regions of interest could be positions with uncertainties of their attribution to an individual person obtained from other, e.g. opti- 
cal imaging techniques or the individual attribution of endogenous compounds within a set of individuals in one sample by automated focusing on level 3 features such as pores. We have recently introduced an MS-based sampling method for the rapid evaluation of latent fingermarks. It circumvents time consuming full MSI scans, by combining rapid image analysis and fast mass spectrometry sampling at discrete positions, which contain the most reliable endogenous chemical information of a fingermark, e.g. at finger pores [16]. Due to the rapid data acquisition scheme conventional sample preparation seems to be the most time-limiting factor in the complete workflow. Therefore, we have applied a method in which we employ sputter deposition of thin gold-layers on top of the latent fingermarks instead of matrix spray coating for MALDI-TOF-MSI sample preparation. Until now, only little is known about the suitability of this preparation method in terms of the application of unsupervised statistical analysis methods to solve the study case of the separation of overlapped fingermarks. To our knowledge there is one publication concerning the usage of multivariate statistical analysis in order to separate overlapped groomed fingermarks by MALDI-TOF-MSI [12]. Although metal-depositionprocesses are usually known as development techniques of latent fingermarks without a chemical interaction and have been recently applied for for laser desorption and ionization (LDI) sample preparation, recent studies indicate interactions between the deposited fingermark surface and the nanomaterials [17] [18] [19]. Based on our MSI results, we will highlight the more surface-science topic from the mass spectrometric point of view. Finally, we introduce and discuss first results for the fast separation of overlapped fingermarks by MS data acquisition on selected $\mathrm{x}, \mathrm{y}$-positions and image reconstitution. Due to the basic character of our study we initially focused on fingermarks prepared under idealized laboratory conditions, which are different to real surfaces observed on crime scene locations.

\section{Results and Discussion}

\subsection{Separation of Overlapped Fingermarks by Statistical Methods}

Nowadays, the collection of high resolution MSI data is in most cases still a very time consuming process, which requires skilled personnel during sample preparation and evaluation of the experimental data. Manual workflows and subjective decisions may influence the result of the overall process, which is a serious limitation particularly in legal processes. In addition, the abilities of new generations of mass spectrometers allow for a significant higher sample throughput even in the field of imaging mass spectrometry. Consequently, sample preparation and data evaluation have to be adapted to these developments. Automation of MSI data processing is highly requested to overcome the current bottlenecks, as well as the application of sophisticated sample preparation techniques. Herein, we have addressed these limitations by the separation of overlapped fingermarks using unsupervised and unbiased statistical analysis methods. For that purpose SciLS lab ${ }^{\mathrm{TM}}$ was used, which is a software solution for the advanced statistical analysis, visualization, and interpretation of MSI data and includes algorithms for unsupervised multivariate analysis, such as spatial segmentation and com- 
ponent analysis to classify cohorts of MS spectra. MSI data were collected by a full MSscan of the overlapping region of a fingermark of two individual donors after normal day life activities, without any further treatment. The fingermarks were deposited on to glass slides initially covered with a $30 \mathrm{~nm}$ Au layer for charge compensation. Thereafter, a $5 \mathrm{~nm}$ Au top layer was deposited and mass spectra were recorded within $24 \mathrm{~h}$ by MSI. The application of the segmentation pipeline in SciLS lab ${ }^{\mathrm{TM}}$ allows for the individualization of the latent fingermarks as depicted in Figure 1. From the 33,794 recorded spectra (337,940 laser shots) 11850 spectra were attributed to the substrate background (35\%). 11,143 (33\%) and 10,801 (32\%) spectra were attributed to the overhead (brown) and the subjacent (green) fingermark, respectively. The delimitation of areas of the different fingermarks is obvious but a closer inspection reveals some limitations: For example, a lack of information of the subjacent fingermark in the overlapping region is apparent. The level 1 topography of the overhead fingermark (brown) was better reproduced than that of the subjacent fingermark (green), which presents artefacts. Level 2 features of the subjacent fingermark are determined by the topological profile of the overhead fingermark. To circumvent the limitation, we have tested several further tools in SciLS lab and have evaluated their qualitative and quantitative performance according fingermark separation.

\subsection{Component Analysis}

Component analysis was performed to discover fundamental components of the MSI data. Component analysis methods are unsupervised processing methods allowing for automatic extraction of underlying trends. The methods decompose the MSI data into score images, which contain main spatial features and component spectra showing the main spectral features (loadings). Results of the first round of component analysis based on the segmentation pipeline limited to 4 components are presented in Figure 2(a).

As indicated, the score image of component 1 contains the main features, which can be attributed to the overlapping fingermarks but without any further discrimination

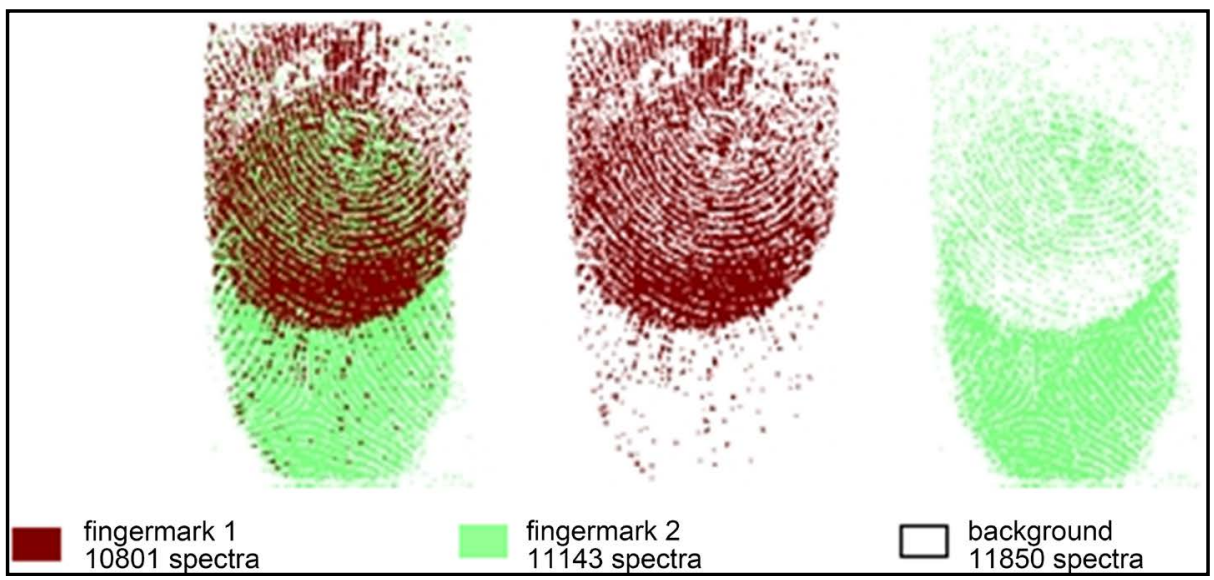

Figure 1. Image result of $S c i L S$ 's segmentation pipeline of overlapped fingermarks. 
of them. Components 2 - 4 show features, which can be associated to the substrate background. In the second round of component analysis the same parameters were used to generate 8 components (Figure 2(b)). In that case the separation of the overlapping fingermarks is obvious from score images of component 1 and 2. A small "shadow" in the overlapping section is still present for these conditions. Enhancing the amount of components to 16 (3rd round, Figure 2(c)), individual fingermarks can be found in score images of component 5 and 12, respectively. As indicated, component

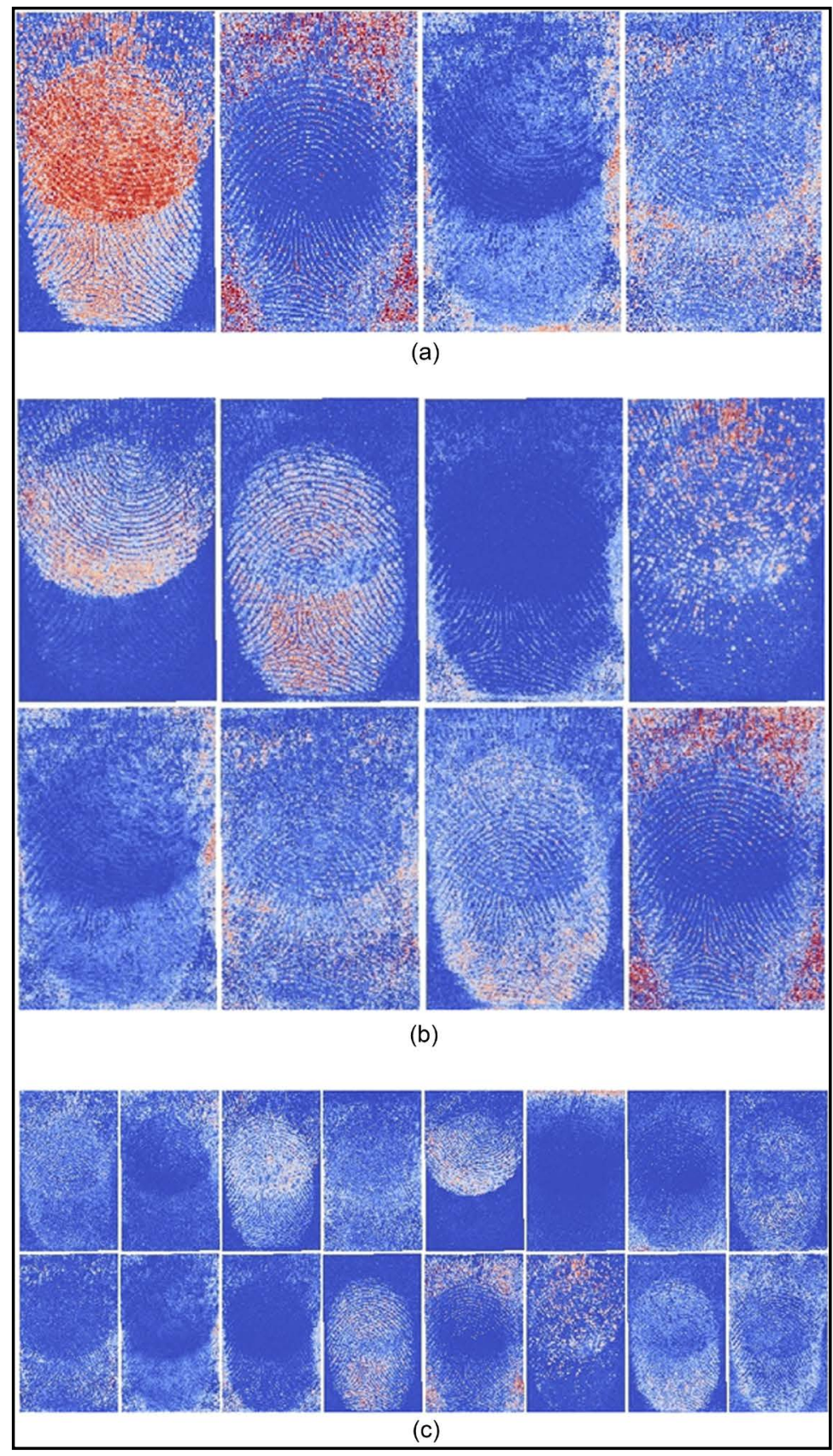

Figure 2. Score images obtained by component analysis in SCiLS lab ${ }^{\mathrm{TM}}$ by attribution of MSI data of the segmentation pipeline to 4,8 , and 16 components as depicted in A, B, C, respectively. 
analysis is suited for the qualitative evaluation of the score images. For a deeper view inside the data set co-localization of markers and Receiver Operating Characteristic (ROC) analysis were performed.

\subsection{Co-Localization of $m / z$-Markers}

Co-localized $\mathrm{m} / \mathrm{z}$ markers are not only of interest for the fingermark differentiation. They also may contain information about the condition of the individual person. Herein, $m / z$-values, which fit best either to the overhead (brown) or the subjacent (green) fingermark obtained by the applied segmentation pipeline were determined by Pearson's correlation analysis, as implemented in the SciLS lab tool "Find Co-Localized $\mathrm{m} / \mathrm{z}$ Values" [20]. The most positive correlated $\mathrm{m} / \mathrm{z}$-regions (markers) by manual adjustment of a threshold (15 regions) are presented in Figure 3, sorted in descending correlation coefficient order. The optical inspection of these single $\mathrm{m} / \mathrm{z}$ images indicates suitable $m / z$ candidates for fingermark differentiation, for instance $m / z=132.6-142.3$; 283.0, 311.1 but also artefacts like 195.9 or 180.0. Artefacts are characterized by a high correlation coefficient at $\mathrm{m} / \mathrm{z}$ positions, where signals are absent. Thus, signal intensities or signal to noise ratios at indicated $\mathrm{m} / \mathrm{z}$ positions have to be taken into consideration for the evaluation process. At this point it has to be stated that one single $\mathrm{m} / \mathrm{z}$ value can in principle be attributed to a large set of potential substances [21]. For example, using the NIST database and performing a search for species data by molecular weight using input data according $\left(\mathrm{m} / \mathrm{z}_{\text {observed }}-1\right) \pm 0.5$ more than 400 hits were found at $\mathrm{m} / \mathrm{z}=$ 132.6 including asparagine or $\mathrm{N}$-glycylglycine $\left(\mathrm{m} / \mathrm{z}_{\text {calc }}=132.05\right)$, as potential indistinguishable endogenous compounds. Using the chemspider database even 3347 compounds were found, which can be limited to 182 compounds using "Metabolic pathways" as data source type, and 25 compounds by additional filtering using the term "Skin". Thus, MS/MS methods have to be taken into consideration for compound identification by their fragments, which is a challenge according the low concentration levels of the secreted endogenous compounds.

Cleaning up the marker candidates from artefacts and comparing the $\mathrm{m} / \mathrm{z}$ intensity

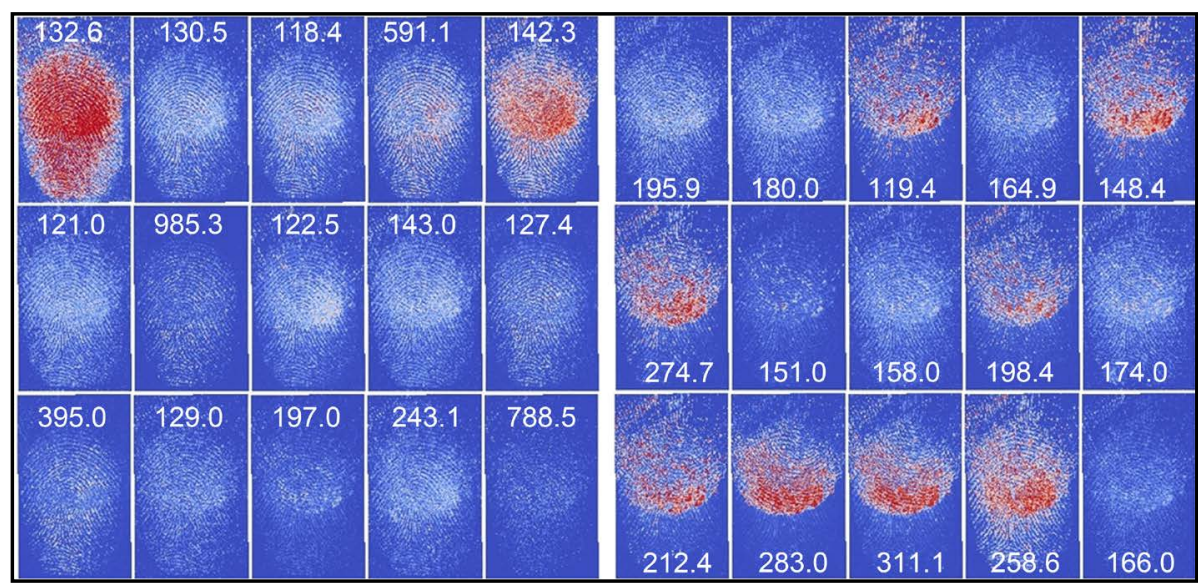

Figure 3. $\mathrm{m} / \mathrm{z}$ images of co-localized markers. 
values of the individual $\mathrm{m} / \mathrm{z}$-images obtained by the Pearsons's analysis against each other by the " $m / z$ Correlation Plot" a correlation matrix is obtained as depicted in Figure 4. Based on the correlation matrix it is possible to find out candidates, which are mostly identical (red) or different (green) to each other. Starting from the most different spectra with the pair of $m / z=985.3 / 198.4$ in the present case $(\mathrm{m} / z$ regions, correlation coefficient $=0.054)$ followed by the most identical spectra of each direction $\mathrm{m} / \mathrm{z}$-pictures can be generated in the order 985.3->394.9->591.0 and 198.4->212.4->274.7, which allow for a more quantitative estimation of the separation efficiency of the individual fingermarks. The correlation matrix clearly reveals correlated, anti-correlated and independent ions. Interestingly, Au clusters are important determinants in the fingermark separation process, e.g. $\mathrm{Au}_{5}, \mathrm{Au}_{2}, \mathrm{Au}_{3}\left(\mathrm{~m} / z_{\text {calc }}=984.8,393.9,590.9\right.$, respectively). Based on these results it can be speculated, that desorption/ionization efficiency of $\mathrm{Au}_{\mathrm{n}}$ depends on the chemical composition of each individual fingermark and the interaction of $\mathrm{Au}$ with endogenous compounds dictates the mass spectrometric behavior. Thus, the approach uses the mass intensity profile of the deposited Au-layer in a nanoscale surface interaction LDI-TOF-MS mode, which is different to conventional matrix systems. The interaction of nanoscale gold and the fingermark is known from multimetal deposition (MMD) and other development techniques. MMD is a very sensitive method for visualizing latent fingermarks and involves the treatment of the fingermark with a gold nanoparticle (AuNP) solution. It is assumed that AuNPs preferentially adsorb to the

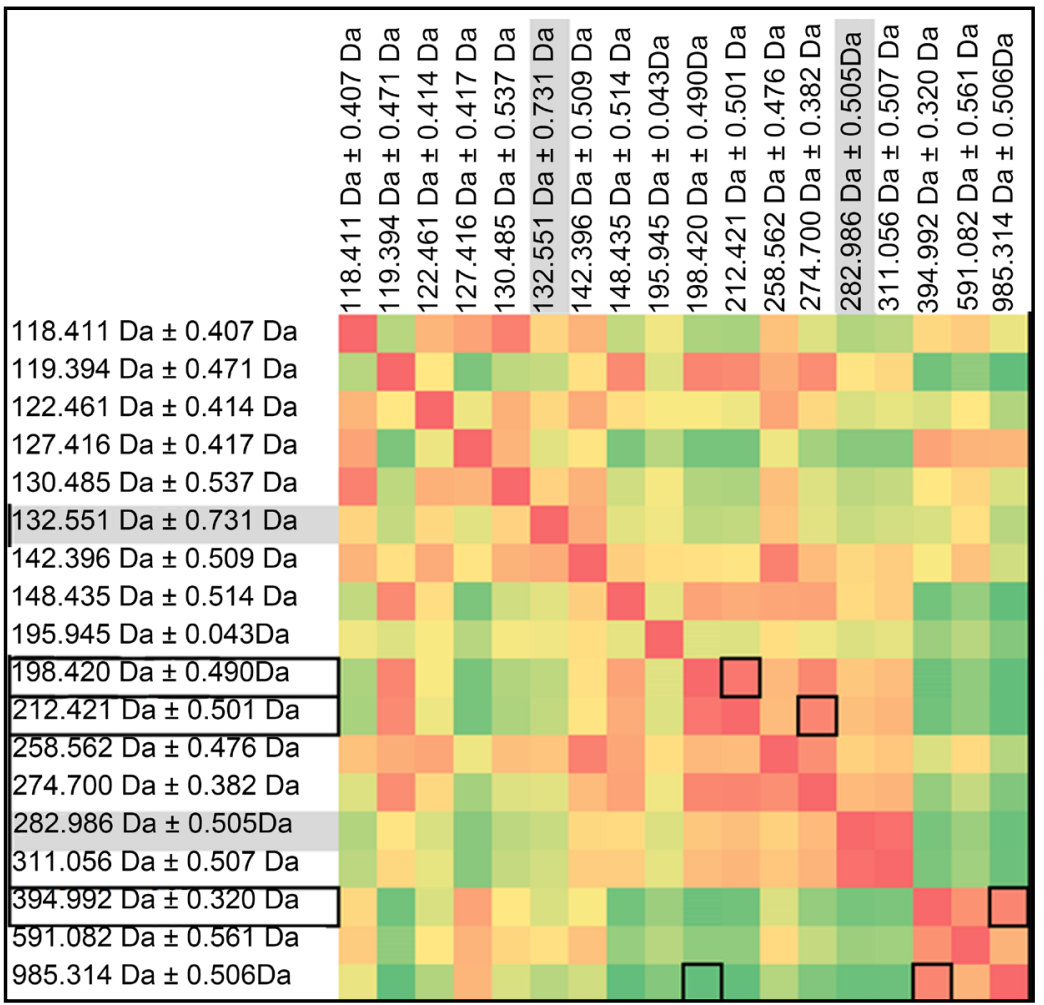

Figure 4. Correlation plot of $\mathrm{m} / \mathrm{z}$-markers. Red: correlated, green: anti-correlated, yellow: neutral. 
fingermark through electrostatic or hydrophobic interactions, and act as a catalytic nucleating site for metallic silver deposited from a developer solution thereafter [22]. Recently, an inverse process was developed, which involves the coating of the substrate instead of the fingermark by the use of surface modified AuNPs in the first step [23] [24].

The mechanism of the interaction of gold nanoparticles with fingermarks is still the subject of current studies [25]. Moreover, the vacuum deposition of different morphologies of gold nanoparticles on the ridges and grooves of the fingermark was reported. Thus, different nanoparticle species may not only be formed by conventional wet chemical processes but also by physical deposition methods and may be influenced by the constitution of the initial surface, e.g. the presence of catalytic or capping chemical surface groups even in a fingermark may play a key role during the generation of different morphologies or amounts of nanoparticles. Although surface influenced nucleation processes are known from physical nanoscale surface modifications such as sputtering, pulsed laser deposition, or molecular beam epitaxy, their impact on the surface modification of samples from biological sources, and their influence on the laser induced desorption and ionization processes, such as present in MSI is largely unexplored [26] [27] [28]. The scanning electron micrographs indicate a different surface nanostructure of vacuum deposited gold of the ridges and grooves regions of a fingermark (Figure 5), which is in accordance with published results [11].

\subsection{ROC-Analysis}

ROC analysis was performed on the results of the segmentation pipeline (class $1=$ fingermark brown; class 2 = fingermark green), to detect further markers which could be suitable to classify the individual fingermarks (Figure 6). Hereby, intensity of a single

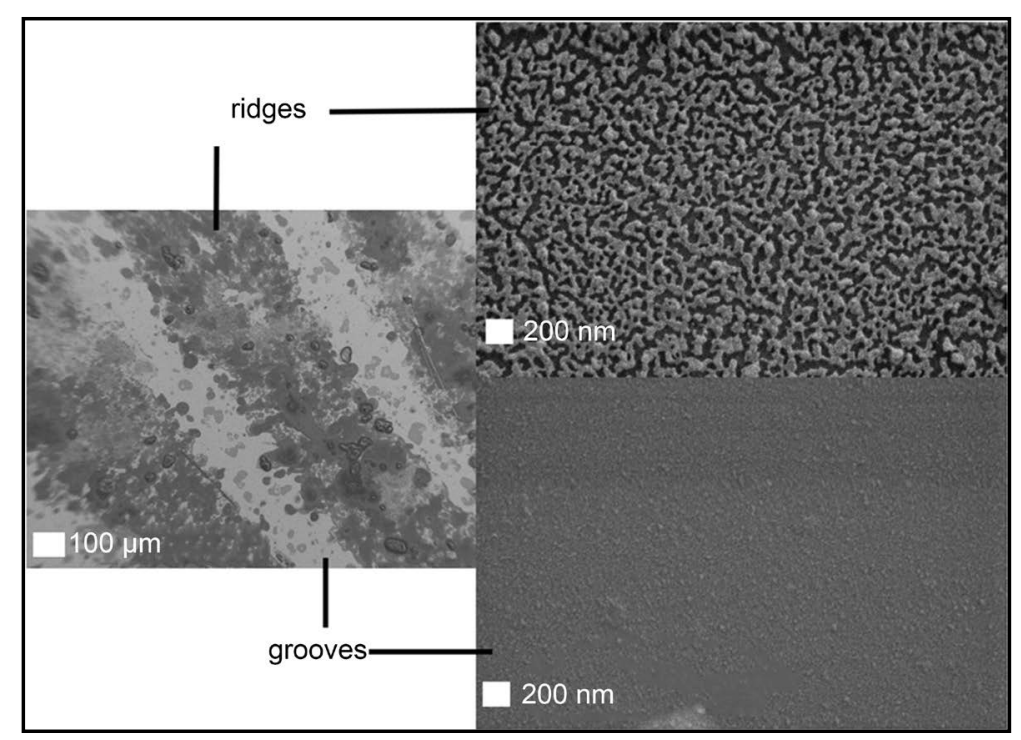

Figure 5. Scanning electron micrograph of a fingermark section. Regions attributed to ridges and grooves are characterized by a different morphology of the deposited Au-layer. 


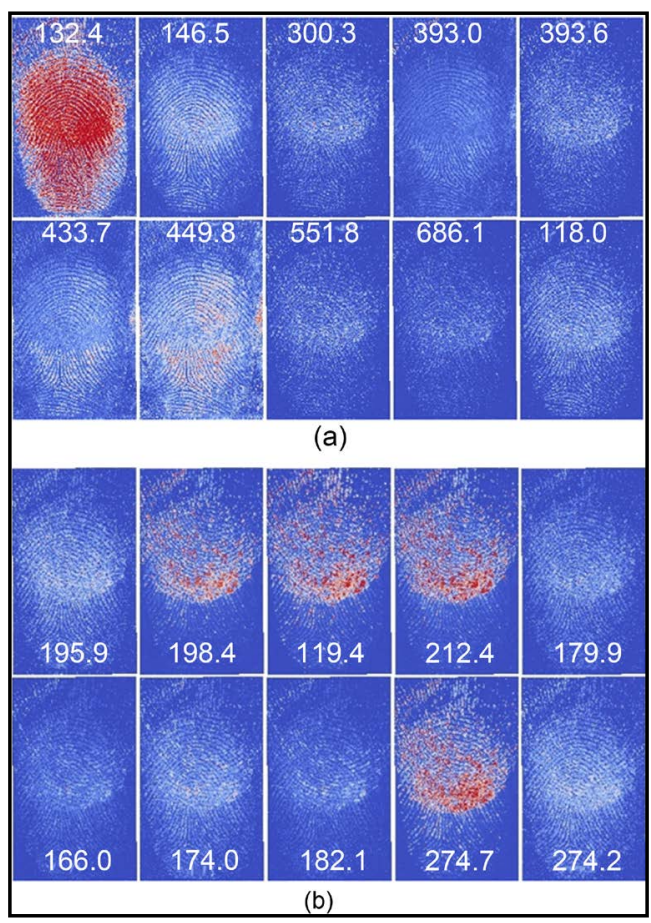

Figure 6. $m / z$ images based on ROC analysis; (a) ROC-threshold $<0.189,10$ best intervals with $\mathrm{m} / \mathrm{z}>100$, sorted according an ascending ROC-value; (b) ROC-threshold $>0.891,10$ best intervals, sorted according a descending ROC-value.

$\mathrm{m} / \mathrm{z}$ value represents the discrimination rule. SciLS lab implemented ROC analysis enables the discovery of compound markers by the comparison of the normalized intensities of $\mathrm{m} / \mathrm{z}$ values in the segmented regions. ROC explores the specificity (false positive rate) and sensitivity (true positive rate) when different intensity thresholds are applied to the $m / z$ values. Discriminating candidates obtained by ROC analysis are specified in Figure 6.

\subsection{Discussion of the Different Processing Methods}

The comparison of the results of the segmentation pipeline with the additional processing of the data by component-, Pearson's-, and ROC-analysis reveals further beneficial information of the individual fingermarks. For the latter ones quantitative statements are possible due to the Pearson's correlation coefficient, correlation matrix results, and ROC-value. Summarizing the results by simple optical inspection of selected $\mathrm{m} / \mathrm{z}$ marker candidates it has to be stated that separated level 2 features appear due to the sophisticated algorithms implemented in SciLS lab, which are not instantly obvious. However, a quantitative estimation of the dependence of different statistical methods on the image quality and their feasibility for database matching algorithms, e.g., a ranking of the generated $\mathrm{m} / \mathrm{z}$ images, is still eligible.

Recently, an algorithm was proposed, which resembles the visual analysis e.g. by an MSI expert, but selects the spatially structured $\mathrm{m} / \mathrm{z}$ images automatically [29]. The core feature of this approach is the ranking of $\mathrm{m} / \mathrm{z}$ images by their level of spatial structure 
by determining the measure of "spatial chaos" in a completely unsupervised manner. The authors have used the approach to measure the presence of known molecules, when their localization in a tissue section is unknown. The individual spatial chaos of each image of a large set of $\mathrm{m} / \mathrm{z}$-images was determined by an image edge detection algorithm and based on the obtained results it was possible to automatically reproduce expert judges. Thus, the approach enables image quality estimation integrated into automatic pipelines.

Since the measure of spatial chaos in a large set of $\mathrm{m} / \mathrm{z}$ images is still not implemented in current commercially available MSI software packages, the "entropy value" of different $\mathrm{m} / \mathrm{z}$ images or image sections was calculated by the use of a MATLAB integrated function, which allows for a quantitative evaluation of the impact of the different statistical methods on the image quality herein [30]. Even in that case the entropy is a statistical measure of randomness/chaos that can be used to characterize the texture of the input image. The determination of the entropy value enables the evaluation of further basic image processing steps that did not rely on statistical $\mathrm{m} / \mathrm{z}$ data processing but on image enhancement methods like contrast adjustment, filtering, and morphological operations, which could be of interest in special forensic cases. Here, the in principle qualitative results obtained by the component analysis can be compared to the results of co-localization studies and ROC analysis in a more quantitative manner. Due to the current image export limitation of the SciLS lab software package (no batch processing) only selected single images were analyzed and processed and the results, including results obtained after image processing steps are listed in Figure 7. Our data show, that the results obtained by co-localization studies have the best impact on the $\mathrm{m} / \mathrm{z}$-image

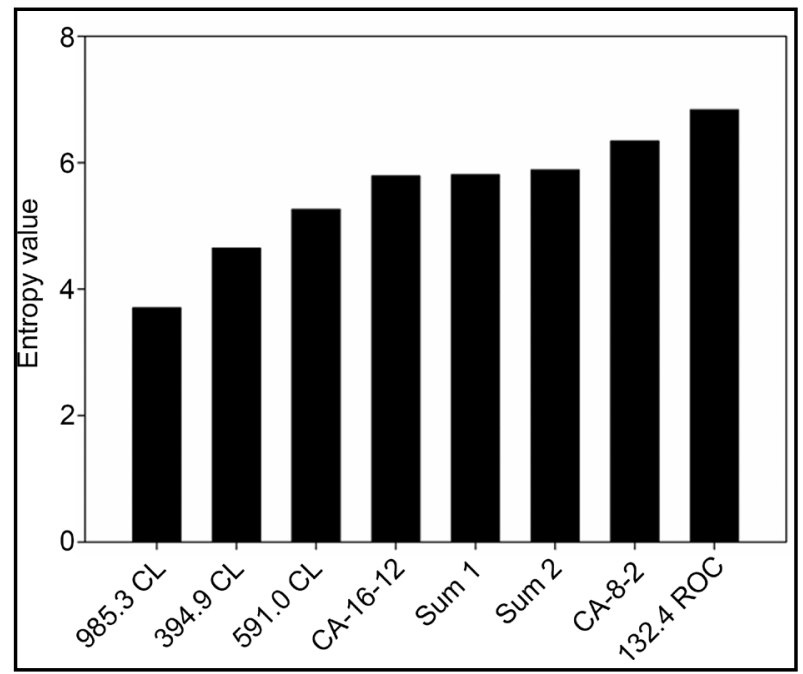

Figure 7. Entropy of selected pictures (previously converted to grayscale images) obtained after different analysis procedures and image processing (CL: co-localization analysis; CA: component analysis (format $\mathrm{CA}-\mathrm{X}-\mathrm{Y}$, where $\mathrm{Y}$ denotes the component of the total components $\mathrm{X}$ with reference to Figure 2); ROC: ROC analysis; Sum 1: Overlay of denoted CL images by addition of the grayscale images; Sum 2: Overlay of the denoted CL images by addition of the grayscale images with $50 \%$ transparency. 
quality based on the calculation of the entropy value. The lowest entropy value of 3.7 was found for the co-localized $\mathrm{m} / z$ value of 985.3 . More interestingly, the entropy values of the $\mathrm{m} / \mathrm{z}$ images obtained from co-localization calculations fit well to the ordered results of the correlation matrix.

Further image processing steps were evaluated according to their influence on the reduction of the image entropy value. The superposition of images from different $\mathrm{m} / \mathrm{z}$ regions in order to reduce noise and enhance dominant fingermark features results in an enhancement of the entropy value. Based on our results we are convinced that the detection of spatial chaos or organized structures in $\mathrm{m} / \mathrm{z}$-images may additionally support MSI processes in fingermark analysis. From the results obtained by statistical $\mathrm{m} / \mathrm{z}$ data processing we conclude that the findings presented herein can, at the first sight, be obtained by handmade analysis by an MSI-expert with manual stepping through each $\mathrm{m} / \mathrm{z}$ value, a proper selection of intervals and visual inspection of the corresponding $\mathrm{m} / \mathrm{z}$ image. However, it has to be stated that automatization, standardization, and quantitative evaluation of the complete detection and separation process of overlapped fingermarks from a full MS-scan has to be the aim and will be necessary to become an accepted process in criminal investigations, law enforcement agencies, or in high-throughput MSI in general. Our contribution herein shows the principal feasibility of such an approach on a single example. The workflow presented herein allows for the generation of marker candidates, which can be used as inputs for database screening e.g. for illicit drugs or pharmaceuticals and may facilitate perpetrator profiling. Future challenges are the identification of substances by MS/MS measurements directly from the fingermark and the evaluation and improvement of the process in real scenarios including database matching. In particular, with regard to this latter aspect, we have successfully matched one fingermark from MSI experiments versus a simple artificial database generated from 3 voluntary test persons $(2 \times 25$ fingermarks, incl. one mirrored set, scanned at $1200 \mathrm{dpi}$, grayscale image). For the generation of the database and matching the "Filterbank-Based Fingermark Matching" program was used in MATLAB [31].

\subsection{Conceptual Approaches for the Rapid MSI Data Acquisition}

In the case of MSI fingermark analysis, a complete MSI scan as presented herein may contain 30\% - 40\% of substrate background scans without any interesting information, which can in principle be reduced. Moreover, the signals obtained from the fingermark itself are redundant. The latter one is even true for the analysis of tissue sections. Recently we have proposed a method, where MS-spectra are recorded only at discrete positions of a fingermark, which may contain the most significant and reliable information about secreted endogenous compounds at the position of the finger pores [16]. For a further development we were interested in the feasibility of the "discrete scanning" concept for the separation of overlapped fingermarks, initially based on blue and red ink covered fingertips for optical comparison reasons. The applied workflow is presented in Figure 8 and consists of the main topics: image acquisition, object detection including large object segmentation, MS control code generation, MS-data acquisition 


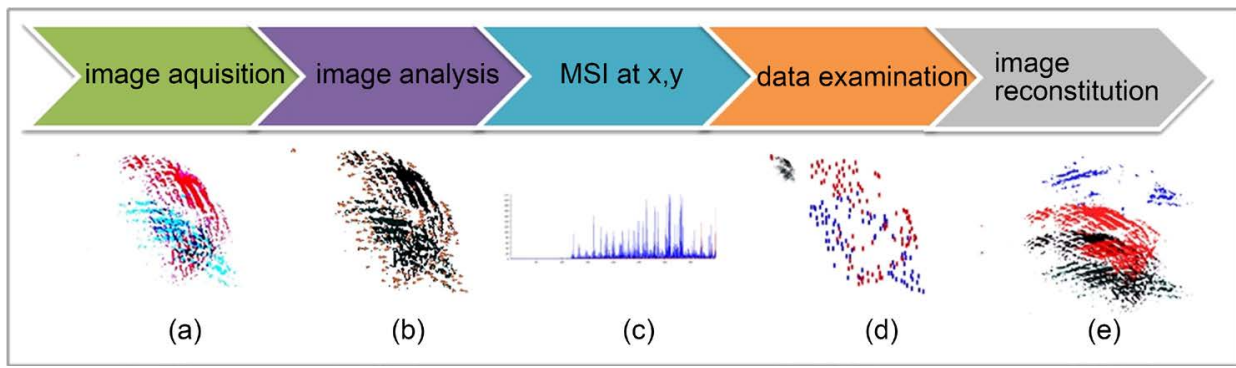

Figure 8. Workflow and results of the separation of overlapped fingermarks by a discrete MSI acquisition schema. (a) Optical microscope image of overlapped fingermarks based on blue- and red-inked fingertips (true color representation). (b) Image analysis and MS control code generation. The orange dots represent the generated $180 \mathrm{x}, \mathrm{y}$-positions (spots) for the laser desorption in the imaging work mode of the mass spectrometer. (c) MS spectra recording at only at the $\mathrm{x}$,y-positions, recording time: $\sim 2.5 \mathrm{~min}$ (at $100 \mathrm{~Hz}$ laser repetition rate, 10 shots/spot). (d) Results of the segmentation analysis (SciLS lab) on the measured spots, the red and blue colored dots represent their attribution to an individual cohort. (e) Reconstructed image of the overlapped fingermark from the individual cohorts (black: overlapped, as represented in (b), red (false color): cohort 1 , corresponds to the red fingerprint in (a), blue (false color): cohort 2, corresponds to the blue fingerprint in (a).

on selected $\mathrm{x}, \mathrm{y}$-positions, unsupervised data examination, and image reconstitution. Since MSI full scan modes may in principle provide $m / z$ images at each $m / z$ value, which can be analyzed by image analysis algorithms, e.g. pattern recognition, entropy determination etc., the fast and discrete MS acquisition process at a set of $\mathrm{x}, \mathrm{y}$-coordinates delivers a scatterplot, where image analysis is not anymore possible. In that case unsupervised statistical methods based on the MS data itself are crucial to obtain any beneficial information. Our first results concerning the concept are presented in Figure 8 and demonstrate the principal feasibility of the approach, although some limitations have been observed, which could be overcome in the future. Mainly, they are attributed to the used object analysis/large object segmentation method during the stage of initial image analysis prior the MS acquisition. For example, large objects may arise from different donors and therefore a suitable segmentation down to level 2 - 3 features is necessary. Certainly, the quality of the reconstituted image can be improved by the use of a more intelligent object segmentation algorithm, e.g. at the first approximation by the object erosion or watershed algorithm as implemented in $M A T L A B$, however, more sophisticated algorithms concerning that topic are under development and investigation in the scientific community [32]. Consequently, the amount of $\mathrm{x}, \mathrm{y}$-positions, which have to be measured increases leading to a so called "Traveling Salesman Problem" during the MSI data acquisition, which has to be considered during the MS control code generation process for the reduction of analysis time. Even for this topic and purpose suitable algorithms are available for implementation [33]. In order to test the true power of the approach the concept has to be further validated and optimized to maximize its reliability in the future.

\section{Summary and Conclusion}

In summary, we have examined different workflows for the unsupervised automated 
analysis of overlapped fingermarks and the assignment of relevant compound markers to their individuals. The determination of spatial chaos in the generated $\mathrm{m} / \mathrm{z}$-images was used to compare the quality and efficiency of the different statistical models. The inclusion of entropy analysis of $m / z$-images may support MSI based fingermark detection approaches. Based on the results of a full scan MSI of fingermarks we introduce a concept of a much faster, data reductive discrete MS acquisition process at selected $\mathrm{x}, \mathrm{y}$-positions based on initial image analysis and reconstitution. Besides fingermark analysis, we claim that the presented workflow with combined optical image and MSI analysis is also applicable to other situations, e.g. the analysis of tissue sections, TLC plates or even particle arrays and may outperform current full scanning modes with respect to the acquisition time and the amount of data significantly. We want to emphasize here that the method does not only work for artificial surfaces but also real surfaces from which the finger marks can be transferred. Latent finger prints can be transferred from the substrate/surface with and conserved on a soft gold sputtered soft membrane (see Materials and Methods section) at low temperatures.

\section{Materials and Methods}

\subsection{Sample Preparation}

Rapid vacuum deposition of a small gold layer on pre-treated glass slides (with a $30 \mathrm{~nm}$ Au-layer) with the deposited fingermark (5 $\mathrm{nm}$ Au-layer) were performed in the chamber of an Auto 306 magnetron sputter coater (Edwards Ltd., United Kingdom) with a distance of $115 \mathrm{~mm}$ between the substrate and the gold-target. The parameters were as follows: operating pressure: $2 \times 10^{-2} \mathrm{mbar}$, power: $30 \mathrm{~W}$, deposition rate 0.24 $\mathrm{nm} \mathrm{s}^{-1}$.

\subsection{Mass Spectrometry Imaging}

Imaging mass spectrometry was performed employing an Autoflex III mass spectrometer equipped with an Nd-YAG SmartBeamR laser (355 nm, Bruker Daltonic, Bremen, Germany). The samples on the slides were mounted via the MTP Slide Adapter II (Bruker Daltonic, Germany). The experiments were performed in reflectron mode by the use of instrument parameters as follows: Ion source $1: 19.11 \mathrm{kV}$, ion source 2:16.65 $\mathrm{kV}$, lens: $8.74 \mathrm{kV}$, reflector: $21.09 \mathrm{kV}$, reflector $2: 9.75 \mathrm{kV}$, detector gain: 1.0 - 5.0, sample rate: 2.0 , shots: 10 , repetition rate: $200 \mathrm{~Hz}$, laser attenuator: offset $75 \%$, range $20 \%$, set1_minimum, fine adjustment: $60 \%$. The mass spectra were acquired in the $\mathrm{m} / \mathrm{z}$ range up to 1000. The instrument was controlled by the FlexControl 3.0 and FlexImaging 2.1 software (Bruker Daltonic, Bremen, Germany). Data were further processed in FlexImaging 2.1 or SciLS lab ${ }^{\mathrm{TM}}$ (Version 2015a, SciLS GmbH, Bremen, Germany). For single spot mass analysis on different objects an image generated by a desktop scanner (EPSON PERFECTION V 300) or stereo microscope (Olympus SZX12 with a ColorView Soft Imaging System) was processed in $M A T L A B$ to obtain their x,y-centroid-coordinates. Centroid coordinates were imported into the mis-File, which controls the run of the mass spectrometer as $\langle$ Spot Pos $=$ " $x, y$ " $\rangle\langle/$ Spot $>$ commands. The same image was used pre- 
viously in FlexImaging for teaching by the use of suitable objects, e.g. laser engraved or printed micro features. Mass analysis was performed using the mode "Tissue Profiling".

\subsection{Image Processing}

Image analysis and partial msi-code generation to control the mass spectrometer was performed in $M A T L A B$. Reconstruction of overlapped ink-based fingermarks based on discrete single spot MS analysis was performed in MATLAB. The indexed image objects with a defined centroid-x,y-spot position were separated due to the exported segmentation map labels calculated in SciLS la $b^{\mathrm{TM}}$. For that reason an appropriate merging of the exported Spot List from FlexImaging and the results from the segmentation map in Excel was necessary to obtain corresponding results.

\subsection{Conservation and Transfer of Latent Finger Prints}

Latent finger prints can be transferred from the substrate/surface with and conserved on a soft gold sputtered soft membrane (Millipore Express plus, Merck KGaA, Germany) at low temperatures.

\subsection{Statement of the Authors and Permission to Use (Anonymized) Data from Human Individuals}

The authors confirm that all experiments (taking finger prints of a volunteer/individual) were performed in accordance with relevant guidelines and regulations of the Leibniz Institute of Surface Modification in Leipzig (Managing board), Germany, the Leibniz Society, and the German Science Foundation (DFG) about experiments with humans and data treatment and protection of personalized data. An explicit informed consent was obtained from the anonymous volunteers providing the finger prints. The individuals explicitly allowed the authors to use the data in the present publication.

\section{Acknowledgements}

The authors thank Mrs. Hertel for the sample preparation by vacuum deposition and A. Prager for the electron microscope images. Partial funding of this work by the Deutsche Forschungsgemeinschaft through SFB 755 and the Leibniz-Gemeinschaft is gratefully acknowledged.

\section{References}

[1] Williams, S.F., et al. (2015) Comparison of the Columnar-Thin-Film and Vacuum-MetalDeposition Techniques to Develop Sebaceous Fingermarks on Nonporous Substrates. Journal of Forensic Sciences, 60, 295-302. https://doi.org/10.1111/1556-4029.12648

[2] Bradshaw, R., et al. (2011) A Novel Matrix-Assisted Laser Desorption/Ionisation Mass Spectrometry Imaging Based Methodology for the Identification of Sexual Assault Suspects. Rapid Communications in Mass Spectrometry, 25, 415-422. https://doi.org/10.1002/rcm.4858

[3] Bradshaw, R., et al. (2013) Spectroscopic Imaging Based Approach for Condom Identification in Condom Contaminated Fingermarks. Analyst, 138, 2546-2557. 
https://doi.org/10.1039/c3an00195d

[4] Benton, M., Chua, M. J., Gu, F., Rowell, F. and Ma, J. (2010) Environmental Nicotine Contamination in Latent Fingermarks from smoker Contacts and Passive Smoking. Forensic Science International, 200, 28-34. https://doi.org/10.1016/j.forsciint.2010.03.022

[5] Girod, A., Ramotowski, R. and Weyermann, C. (2012) Composition of Fingermark Residue: A Qualitative and Quantitative Review. Forensic Science International, 223, 10-24. https://doi.org/10.1016/j.forsciint.2012.05.018

[6] He, Y., et al. (2014) Immunological Multimetal Deposition for Rapid Visualization of Sweat Fingerprints. Angewandte Chemie International Edition, 53, 12609-12612.

[7] Bailey, M.J., et al. (2015) Rapid Detection of Cocaine, Benzoylecgonine and Methylecgonine in Finger Prints Using Surface Mass Spectrometry. Analyst, 140, 6254-6259. https://doi.org/10.1039/C5AN00112A

[8] Hazarika, P. and Russell, D.A. (2012) Advances in Fingerprint Analysis. Angewandte Chemie International Edition, 51, 3524-3531. https://doi.org/10.1002/anie.201104313

[9] Lauzon, N., Dufresne, M., Chauhan, V. and Chaurand, P. (2015) Development of Laser Desorption Imaging Mass Spectrometry Methods to Investigate the Molecular Composition of Latent Fingermarks. Journal of the American Society for Mass Spectrometry, 26, 878886. https://doi.org/10.1007/s13361-015-1123-0

[10] Francese, S., et al. (2013) Beyond the Ridge Pattern: Multi-Informative Analysis of Latent Fingermarks by MALDI Mass Spectrometry. Analyst, 138, 4215-4228. https://doi.org/10.1039/c3an36896c

[11] Tang, H.-W., Lu, W., Che, C.-M. and Ng, K.-M. (2010) Gold Nanoparticles and Imaging Mass Spectrometry: Double Imaging of Latent Fingerprints. Analytical Chemistry, 82, 1589-1593. https://doi.org/10.1021/ac9026077

[12] Bradshaw, R., Rao, W., Wolstenholme, R., Clench, M.R., Bleay, S. and Francese, S. (2012) Separation of Overlapping Fingermarks by Matrix Assisted Laser Desorption Ionisation Mass Spectrometry Imaging. Forensic Science International, 222, 318-326. https://doi.org/10.1016/j.forsciint.2012.07.009

[13] Bright, N.J., et al. (2012) Determination of the Deposition Order of Overlapping Latent Fingerprints and Inks Using Secondary Ion Mass Spectrometry. Analytical Chemistry, 84, 4083-4087. https://doi.org/10.1021/ac300185j

[14] Attard Montalto, N., et al. (2014) Determining the Chronology of Deposition of Natural Fi Ngermarks and Inks on Paper Using Secondary Ion Mass Spectrometry. Analyst, 139, 4641-4653. https://doi.org/10.1039/C4AN00811A

[15] Qian, K., Schott, M., Zheng, W. and Dittmann, J. (2014) Context-Based Approach of Separating Contactless Captured High-Resolution Overlapped Latent Fingerprints. IET Biometrics, 3, 101-112. https://doi.org/10.1049/iet-bmt.2013.0057

[16] Elsner, C. and Abel, B. (2014) Ultrafast High-Resolution Mass Spectrometric Finger Pore Imaging in Latent Finger Prints. Scientific Reports, 4, 6905. https://doi.org/10.1038/srep06905

[17] Choi, M.J., McDonagh, A.M., Maynard, P. and Roux, C. (2008) Metal-Containing Nanoparticles and Nano-Structured Particles in Fingermark Detection. Forensic Science International, 179, 87-97. https://doi.org/10.1016/j.forsciint.2008.04.027

[18] Sekuła, J., Nizioł, J., Rode, W. and Ruman, T. (2015) Gold Nanoparticle-Enhanced Target (AuNPET) as Universal Solution for Laser Desorption/Ionization Mass Spectrometry Analysis and Imaging of Low Molecular Weight Compounds. Analytica Chimica Acta, 875, 61-72. https://doi.org/10.1016/j.aca.2015.01.046 
[19] Nizioł, J., Rode, W., Zieliński, Z. and Ruman, T. (2013) Matrix-Free Laser DesorptionIonization with Silver Nanoparticle-Enhanced Steel Targets. International Journal of Mass Spectrometry, 335, 22-32. https://doi.org/10.1016/j.ijms.2012.10.009

[20] McDonnell, L. a., Van Remoortere, A., Van Zeijl, R. J. M. and Deelder, A. M. (2008) Mass Spectrometry Image Correlation: Quantifying Colocalization. Journal of Proteome Research, 7, 3619-3627. https://doi.org/10.1021/pr800214d

[21] Winkler, R. (2015) SpiderMass: Semantic Database Creation and Tripartite Metabolite Identification Strategy. Journal of Mass Spectrometry, 50, 538-541. https://doi.org/10.1002/jms.3559

[22] Becue, A., Champod, C. and Margot, P. (2007) Use of Gold Nanoparticles as Molecular Intermediates for the Detection of Fingermarks. Forensic Science International, 168, 169-176. https://doi.org/10.1016/j.forsciint.2006.07.014

[23] Jaber, N., et al. (2012) Visualization of Latent Fingermarks by Nanotechnology: Reversed Development on Paper-A Remedy to the Variation in Sweat Composition. Angewandte Chemie, 51, 12224-12247. https://doi.org/10.1002/anie.201205259

[24] Shenawi, S., Jaber, N., Almog, J. and Mandler, D. (2013) A Novel Approach to Fingerprint Visualization on Paper Using Nanotechnology: Reversing the Appearance by Tailoring the Gold Nanoparticles' Capping Ligands. Chemical Communications, 49, 3688-3690. https://doi.org/10.1039/c3cc41610k

[25] Moret, S., Bécue, A. and Champod, C. (2014) Nanoparticles for Fingermark Detection: An Insight into the Reaction Mechanism. Nanotechnology, 25, Article ID: 425502. https://doi.org/10.1088/0957-4484/25/42/425502

[26] Malinský, P., Slepička, P., Hnatowicz, V. and Švorčík, V. (2012) Early Stages of Growth of Gold Layers Sputter Deposited on Glass and Silicon Substrates. Nanoscale Research Letters, 7, 241. https://doi.org/10.1186/1556-276X-7-241

[27] Siegel, J., et al. (2012) Structural, Electrical and Optical Studies of Gold Nanostructures Formed by Ar Plasma-Assisted Sputtering. Nuclear Instruments and Methods in Physics Research Section B, 272, 193-197. https://doi.org/10.1016/j.nimb.2011.01.063

[28] Švorčík, V., et al. (2006) Characterization of Evaporated and Sputtered Thin Au Layers on Poly(Ethylene Terephtalate). Journal of Applied Polymer Science, 99, 1698-1704. https://doi.org/10.1002/app.22666

[29] Alexandrov, T. and Bartels, A. (2013) Testing for Presence of Known and Unknown Molecules in Imaging Mass Spectrometry. Bioinformatics, 29, 2335-2342. https://doi.org/10.1093/bioinformatics/btt388

[30] http://de.mathworks.com/help/images/ref/entropy.html

[31] https://de.mathworks.com/matlabcentral/fileexchange/4239-fingerprint-recognition-system /content/rel51/codice b.m

[32] Zhang, C., Sun, C., Su, R. and Pham, T.D. (2015) Clustered Nuclei Splitting via Curvature Information and Gray-Scale Distance Transform. Journal of Microscopy, 259, 36-52. https://doi.org/10.1111/jmi.12246

[33] Laporte, G. (1992) The Vehicle Routing Problem: An Overview of Exact and Approximate Algorithms. European Journal of Operational Research, 59, 345-358. https://doi.org/10.1016/0377-2217(92)90192-C 
Submit or recommend next manuscript to SCIRP and we will provide best service for you:

Accepting pre-submission inquiries through Email, Facebook, LinkedIn, Twitter, etc. A wide selection of journals (inclusive of 9 subjects, more than 200 journals)

Providing 24-hour high-quality service

User-friendly online submission system

Fair and swift peer-review system

Efficient typesetting and proofreading procedure

Display of the result of downloads and visits, as well as the number of cited articles

Maximum dissemination of your research work

Submit your manuscript at: http://papersubmission.scirp.org/

Or contact aces@scirp.org 